\title{
ESTIMATING RECOVERY TIME OF THE PLANKTON: EVIDENCE FROM MACROEVOLUTIONARY PATTERNS IN THE CENOZOIC PLANKTONIC FORAMINIFERA
}

\author{
ARNOLD*, Anthony J. and W.C. Parker, Department of Geology, Florida State University, \\ Tallahassee, FL 32333
}

The recovery of biological populations from ecological disasters can be monitored by examining the age structure of the recovering population. Immediately after a collapse, the age distribution of the population tends to shift toward an increase in the proportion of juvenile individuals.

In this work, we explore a similar phenomenon at the macroevolutionary level in the hopes that we might measure the time required for restabilization of the species longevity distribution of the planktonic foraminifera after the $\mathrm{K} / \mathrm{T}$ event. Because influences such as density dependence, carrying capacity, and organismal senescence cannot be assumed to have analogues at the species level, we must take an approach that explores rather than assumes the extent of the hierarchical similarity between organisms and species.

In order to compare the realized species longevity distribution during the $\mathrm{K} / \mathrm{T}$ recovery with modelled expectations, it is first necessary to determine the expected species longevity distribution. Since the Cenozoic planktonic foraminifera include extant ("censored") species for which the full longevity is unknown, we must perform a correction in order to characterize the expected ideal, uncensored longevity distribution. This requires that we make certain assumptions about the form (but not the parameters) of the longevity distribution. The exponential and the Weibull survivorship models allow us to calculate the corrected longevity distribution under assumptions of longevity-independent and longevity-dependent extinction probabilities, respectively.

We used these calculated longevity distributions to run Monte-Carlo simulations of the recovery using the assumptions of random origination and density-dependent origination. Mean longevity at 1 my intervals was calculated, and $95 \%$ confidence intervals determined. Recovery from the $\mathrm{K} / \mathrm{T}$ extinction was estimated from the time required to achieve a stable mean longevity.

In terms of their effect on estimated time to recovery, there was no significant difference between the exponential and Weibull models; both yielded a predicted time to recovery of approximately $15 \mathrm{my}$, which agrees well with the realized time to recovery. Significant deviations from the predicted pattern of recovery are seen in the Paleocene and late Neogene and may be related to temporal variation in origination rates of short-lived species. 